Recepción: 12 / 04 / 2018

Aceptación: 08 / 05 / 2018

Publicación: 02 / 07 / 2018

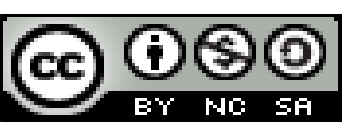

Ciencias de la educación

\title{
La investigación como acción participativa en la educación superior
}

\author{
Research as participatory action in higher education
}

Pesquisa como ação participativa no ensino superior

\author{
Lucia G. Chávez-Ruano ${ }^{\mathrm{I}}$ \\ luciachavez@gmail.com
}

\section{Correspondencia: luciachavez@gmail.com}

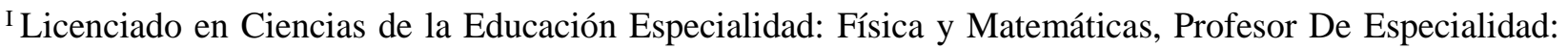
Física y Matemáticas, Magister en Desarrollo del Pensamiento y la Educación, Docente de la Universidad "Luis Vargas Torres” Esmeraldas, Ecuador.
\end{abstract}




\section{Resumen}

La humanidad se desarrolló a través de la investigación y por lo cual es fundamental para el crecimiento de las sociedades. El conocimiento, generado por las investigaciones, debe ser fomentado por un entorno social. Es por ello que, la acción participativa de la comunidad universitaria, en la investigación, fomentara aún más al desarrollo del conocimiento y por ende el desarrollo de la sociedad. Para cumplir con este desarrollo se deben quebrar viejos paradigmas dentro de las investigaciones universitarias, uno de las principales es que solo deben ser realizadas por científicos especializados. El objetivo principal es analizar la investigación como acción participativa en la educación superior. El proceso metodológico se basó en revisión de textos, documentos y artículos científicos publicados disponibles en la web. Los resultados obtenidos arrojaron que la investigación debe ser participativa y protagónica dentro de las instituciones de educación superior donde cada miembro puede plantear ideas, sugerencias y soluciones desde sus experiencias en el ámbito social y cultural. Como conclusión, se determinó que para poder lograr la investigación participativa se debe masificar, desde todos los vértices, la educación superior generando así una inversión en conocimientos, derivados de las investigaciones, que serán extrapolados al desarrollo productivo y social del país.

Palabras clave: Investigación; conocimiento; participación; paradigma; masificación.

\section{Abstract}

Humanity was developed through research and therefore is essential for the growth of societies. Knowledge, generated by research, must be fostered by a social environment. That is why, the participatory action of the university community, in research, will further promote the development of knowledge and therefore the development of society. In order to comply with this development, old paradigms within university research must be broken, one of the main ones is that they should only be carried out by specialized scientists. The main objective is to analyze research as participatory action in higher education. The methodological process was based on the revision of texts, documents and scientific articles published on the web. The results obtained showed that research should be participatory and protagonist within higher education institutions where each member can present ideas, suggestions and solutions from their experiences in the social and cultural field. As a conclusion, it was determined that in order to achieve participatory research, higher education should be massified, from all the vertices, generating an investment in 
knowledge, derived from research, which will be extrapolated to the productive and social development of the country.

Keywords: Research; knowledge; participation; paradigm; overcrowding.

\section{Resumo}

A humanidade foi desenvolvida através da pesquisa e, portanto, é essencial para o crescimento das sociedades. O conhecimento, gerado pela pesquisa, deve ser estimulado por um ambiente social. Por isso, a ação participativa da comunidade universitária, em pesquisa, promoverá ainda mais o desenvolvimento do conhecimento e, portanto, o desenvolvimento da sociedade. A fim de cumprir com esse desenvolvimento, velhos paradigmas dentro da pesquisa universitária devem ser quebrados, um dos principais é que eles só devem ser realizados por cientistas especializados. O objetivo principal é analisar a pesquisa como ação participativa no ensino superior. O processo metodológico baseou-se na revisão de textos, documentos e artigos científicos publicados na web. Os resultados obtidos mostraram que a pesquisa deve ser participativa e protagonista nas instituições de ensino superior, onde cada membro pode apresentar idéias, sugestões e soluções a partir de suas experiências no campo social e cultural. Como conclusão, determinou-se que, para a realização da pesquisa participativa, o ensino superior deveria ser massificado, a partir de todos os vértices, gerando um investimento em conhecimento, derivado da pesquisa, que será extrapolado para o desenvolvimento produtivo e social do país.

Palavras chave: Pesquisa; conhecimento; participação; paradigma; superlotação.

\section{Introducción}

El desarrollo del hombre en su entorno va enmarcado, desde la edad de piedra, por una constante curiosidad involucrada con las necesidades básicas. Satisfacer estas necesidades han permitido al hombre evolucionar y conocer nuevas formas de crear a través del ensayo y error. Estos nuevos aprendizajes pasaban de generación en generación donde cada uno aportaba alguna mejora obteniendo así resultados más rápidos y fidedignos. Todos estos nuevos aprendizajes es producto de una investigación personal y grupal donde cada miembro de la sociedad participa generando desde sus experiencias, creencias y cultura, un conocimiento que a la medida del tiempo será cambiante. La investigación como factor fundamental en el desarrollo del conocimiento tiene, en 
su columna vertebral, la chispa de la curiosidad para generar sociedades con nuevos propósitos para lograr sus objetivos.

Las instituciones de educación superior son un baluarte en el desarrollo de investigaciones. Este desarrollo se ha venido estableciendo por mecanismos que solo satisfacen las necesidades individuales, con trabajos de grado que quedan almacenados en los estantes de las bibliotecas sin poder ser ejecutados, con trabajos de ascenso que buscan garantizar una mejor posición dentro de las instituciones e investigaciones científicas que solo buscan el renombre del investigador o institución sin indagar, generar controversia o buscar nuevas soluciones a los problemas planteados. Todos estos factores terminan por llevar a la investigación a un sistema prácticamente burocrático. No solo se convierte en requisito para ser reconocido, sino que también se transforma en un paradigma que se caracteriza por ser "elitista y tecnocrático".

Este tipo de paradigma va enmarcado en el desarrollo metodológico, ya que, se vuelve desigual entre los agentes que conforman la investigación. "En éste el papel dominante corresponde al cliente, los técnicos adoptan una función meramente instrumental y los destinatarios se sitúan en una posición dependiente y pasiva, siendo meros receptáculos de una trama movida por ajenos intereses" (Pereda, 1993, p. 1-2).

Todos estos factores se asemejan al proceso original de la comunicación donde hay un emisor y receptor en un solo sentido. No se toma en cuenta que la comunicación debe ser en feed-back entre ese emisor y receptor. "El conocimiento que se genera sea útil socialmente, productivo en lo científico y generador a la vez de alternativas viables a ese principio de realidad que hace que nuestros países sigan siendo tan asimétricos, tan inexcusable e inmoralmente injustos" (Sánchez, 2007). Lo cual determinada que el paradigma en la investigación sigue tomando un papel fundamental en el desarrollo del conocimiento, donde el papel lo lleva una persona clasificada científicamente para tal caso.

Para enfrentar este dilema se debe abrir espacios donde la investigación sea un todo, desde la misma generación de conocimientos como a los actores que promueven tal evento. Esto sucede gracias a una relación eficacia-proceso de enseñanza la cual debe ser trasmitida a un entorno social fundamental, ya que, los miembros de la sociedad poseen conocimientos adquiridos durante la vida los cuales brindan alto valor para el desarrollo de la investigación. No solo la 
investigación puede ser realizada a través de métodos científicos, también es importante demostrar que la sociedad puede participar en el desarrollo del conocimiento con el fin de garantizar el progreso de un país.

Ahora ese entorno social se puede trasladar al entorno dentro de las instituciones de educación superior donde la generación de investigaciones debe ser más profunda, ya que, los miembros de la comunidad universitaria poseen mayores conocimientos de ciertas áreas a las cuales se pueda desarrollar una investigación. Estas experiencias enmarcadas por el contacto con experiencias académicas, ayudado con los equipos e instrumentos de los laboratorios fomentan el desarrollo del conocimiento. "El papel del agente educativo no es el de enseñar al grupo como proponer soluciones y/o tareas, sino aprovechar sistemáticamente lo que el grupo o miembros del grupo proponen" (Gajardo, 1983, p.14). Por supuesto, el agente educativo es la misma institución de educación superior que debe impulsar la generación de investigación entre todos los miembros de la comunidad universitaria.

Por lo tanto, este artículo tiene como objetivo generar un análisis sobre la investigación como acción participativa en la educación superior. Para lograr la consecución de este planteamiento se realizaron diferentes investigaciones como revisión de textos, documentos y artículos científicos publicados disponibles en la web y sobre todo entrevistas con los miembros de la comunidad universitaria.

\section{Método}

El desarrollo de este trabajo se basó en el estudio y análisis de la investigación como acción participativa entre todos los miembros que conforman las instituciones de educación superior debido a que sus experiencias, desde varios puntos de vista, pueden generar diferentes aristas de la investigación y por lo tanto un mayor crecimiento del conocimiento. Esto significa darles valor agregado a los conocimientos adquiridos por el personal docente, técnico de laboratorio, obreros, administrativos y estudiantes con el fin de generar investigaciones que puedan ser desarrolladas en procesos industriales y poder así ser partícipes en el desarrollo del país.

La metodología se basó en la revisión de textos, documentos y artículos científicos publicados disponibles en la web. Aunado a esto también se aplicaron entrevistas a los miembros de la 
comunidad universitaria para conocer cuáles son los factores que pueden impulsar el desarrollo participativo de la investigación.

\section{Resultados}

El proceso evolutivo del hombre va enmarcado con el desarrollo constante de la investigación. Este desarrollo es generado por una "curiosidad" que está determinada por la satisfacción de las necesidades básicas. Al principio el hombre buscaba la manera de encontrar esas soluciones a través de métodos ordinarios o de ensayo y error. Con ello fue desarrollando su perspectiva de la realidad, siendo más analítico y pedagógico para poder trasmitir sus ideas.

"El hemisferio izquierdo, que controla el habla, la escritura y la habilidad matemática, tiene la modalidad de pensamiento racional y lógico, por lo cual al estudiar los problemas planteados procede paso a paso mediante un análisis riguroso" (Pérez-Rubin, 2001, p. 9). Es así como el hombre desarrollo sus habilidades para poder entonces solucionar los problemas más habituales en su entorno y así empezar a manifestarse a través de la comunicación entre las personas, sean por diferentes medios, hablado o escrito.

Este desarrollo intuitivo de la investigación no es más que un sinónimo de la investigación por descubrimiento la cual produce ciertos valores negativos de los especialistas en la materia.

"El movimiento de aprendizaje por descubrimiento no puede despacharse -como a menudo hemos hecho- con una simple referencia a sus negativos resultados, a su fracaso en favorecer un aprendizaje de las ciencias más efectivo, a la denuncia de su inductivismo extremo, falta de atención a los contenidos, etc" (Gil Pérez, 1994, p. 3).

Estos factores negativos no pueden opacar el desarrollo de la investigación a través de descubrimientos que han favorecido a la humanidad desde sus comienzos. Cada investigación ha generado un completo desarrollo de las naciones desde el punto de vista económico, social y cultural. "No hay un patrón universal de desarrollo, éste difiere de una sociedad a otra. La cultura de los pueblos y sus historias tiene un sitial importante en ello" (Valcárcel, 2006). Es allí donde la investigación va a depender de las necesidades que estén en el entorno y como poder superar las expectativas generadas por la sociedad. 
Indistintamente de que la investigación sea formulada por descubrimientos o pautada por algoritmos científicos es importante resaltar un buen desarrollo metódico para ir cubriendo las etapas que vayan saliendo en el desarrollo de la misma. Todo esto conlleva a solucionar el problema de ambigüedades o sustentos bajos de conocimiento

Para realizar un trabajo de investigación es necesario llevar a cabo un desarrollo metódico que permita la adecuada consecución de los objetivos propuestos, así como una formulación clara, concreta y precisa del problema y una metodología de investigación rigurosa y adecuada al tipo de trabajo (López Noguero, 2002, p. 1)

Todos estos pasos también generan ciertas interrogantes que van a depender de los actores que están involucrados debido a que se abren paradigmas científicos que se relacionan sobre qué tipo de personas pueden realizar la investigación. Estos paradigmas dependen de los criterios de eficacia, como juzgar el éxito de una persona cuando realizo la tarea, y los criterios de enseñanza, como enfoque de conductas personales al momento de realizar la investigación. "Es de algún modo paradójico que los estados internos cognitivo y afectivo, tanto en los educandos como los educadores, reciban igual peso que las acciones observables de ambos" (Wittrock, 1989, p. 8). Todo esto lleva a colación la importancia que tiene la relación de la eficacia con el procedimiento de enseñanza para poder producir una buena investigación.

"La universidad debe fomentar el espíritu de investigación en todos los niveles de la formación académica, propiciando la formación integral de sus estudiantes de pregrado y postgrado, con la participación activa del claustro de profesores" (Carizo Estévez, 2010, p. 3). Este tipo de investigación en los niveles planteados es lo que se ha fomentado siempre ayudando aún más a crecer el paradigma de quienes son las personas acordes para la realización de la investigación. La investigación, en el entorno de la educación, siempre ha tenido como actor principal al docente sin tomar en cuenta la experiencia de técnicos de laboratorio, personal obrero y administrativo, y también las experiencias de los estudiantes. Este tipo de forma es el principal paradigma incrustado en el colectivo universitario, sin valorar los saberes propuestos por los demás miembros de la comunidad los cuales representan un baluarte significativo para la generación de conocimientos a través de la investigación. 
Aunado a esto, uno de los factores que puede impulsar la investigación cooperativa dentro del claustro universitario es la expansión que han tenido las universidades en los últimos años la cual ha sido de gran importancia para el crecimiento de las investigaciones, como se ve en la Figura 1.

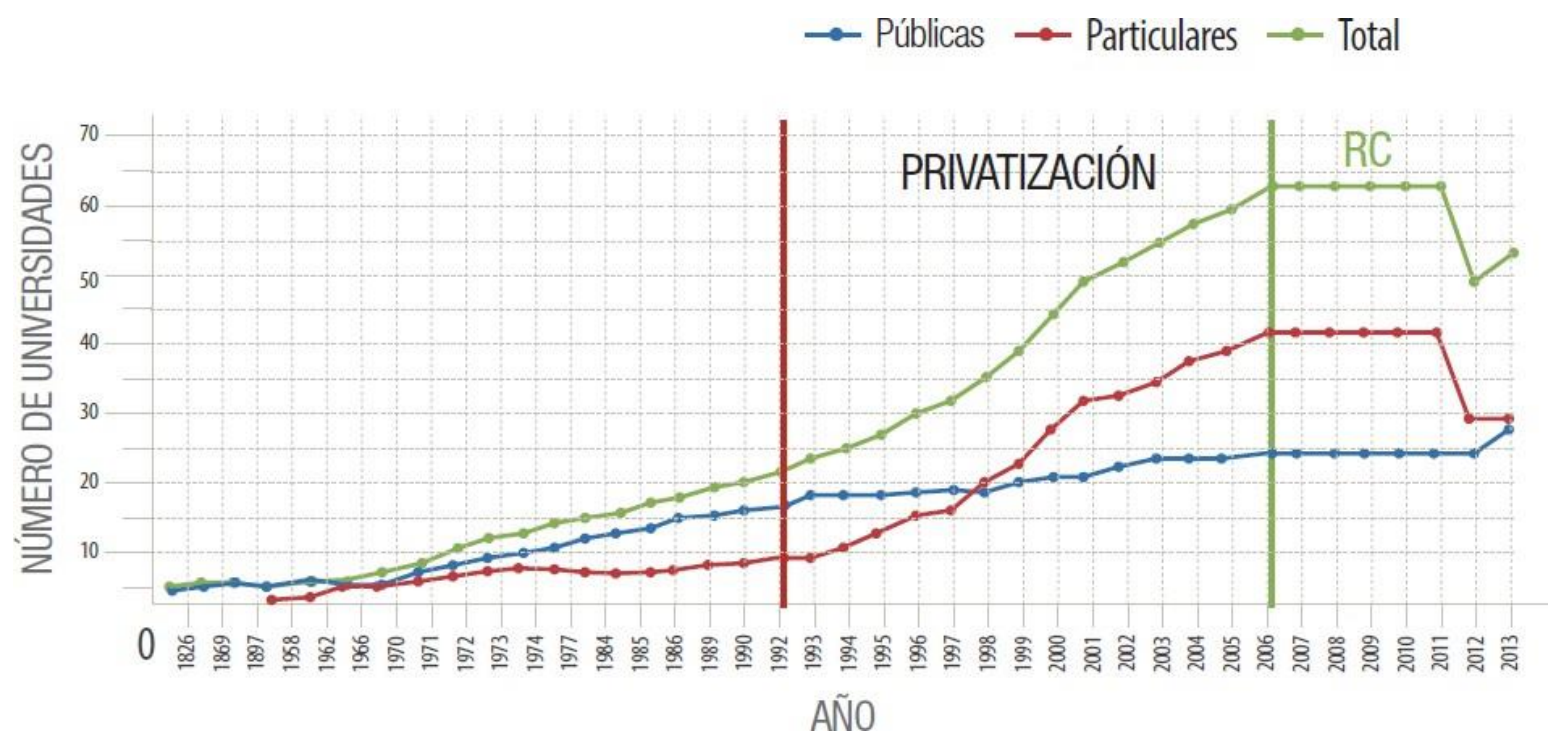

Figura 1. Número de universidades según año de creación en Ecuador.

Fuente: (Ramírez Gallegos, 2013, p. 38).

"La masificación de la educación superior es el producto de la dinámica social particular, en la cual la consolidación de amplios sectores medios se tradujo en una creciente demanda educativa en el nivel superior" (Gallo, 2005, p. 14), lo cual permite que los sectores sociales, productivos y culturales se adjunten a la dinámica de la investigación fomentando el desarrollo de investigaciones.

Es importante resaltar el juego que hace la tecnología en acercar estos actores dentro de las universidades y es por ello que las herramientas como las TIC (Tecnologías de la Información y la Comunicación) son un paso adelante para esa masificación en la investigación.

El impacto de las nuevas tecnologías por su parte, está contribuyendo a la globalización de la educación, permitiendo acortar las distancias, expandir la educación transfronteriza y las modalidades de educación en red, y al generar la educación virtual, viabilizar nuevas prácticas pedagógicas de simulación, de autoaprendizaje y de praxis, y una educación no presencial (Rama, 2006, p. 4). 
Esta masificación de la educación desde todos los aspectos, tanto de aumento de matrícula como de las herramientas tecnológicas, produce un incremento del gasto público de la nación, como se puede observar en la Figura $\mathrm{N}^{\circ}$ 2. Este incremento del gasto público puede verse como una apuesta al desarrollo de investigaciones con el fin de resolver situaciones reales desde todos los ámbitos del país.

\section{$\longrightarrow$ Gastos Públicos ES (nominal) $\quad \longrightarrow$ Gastos Públicos \% PIB}

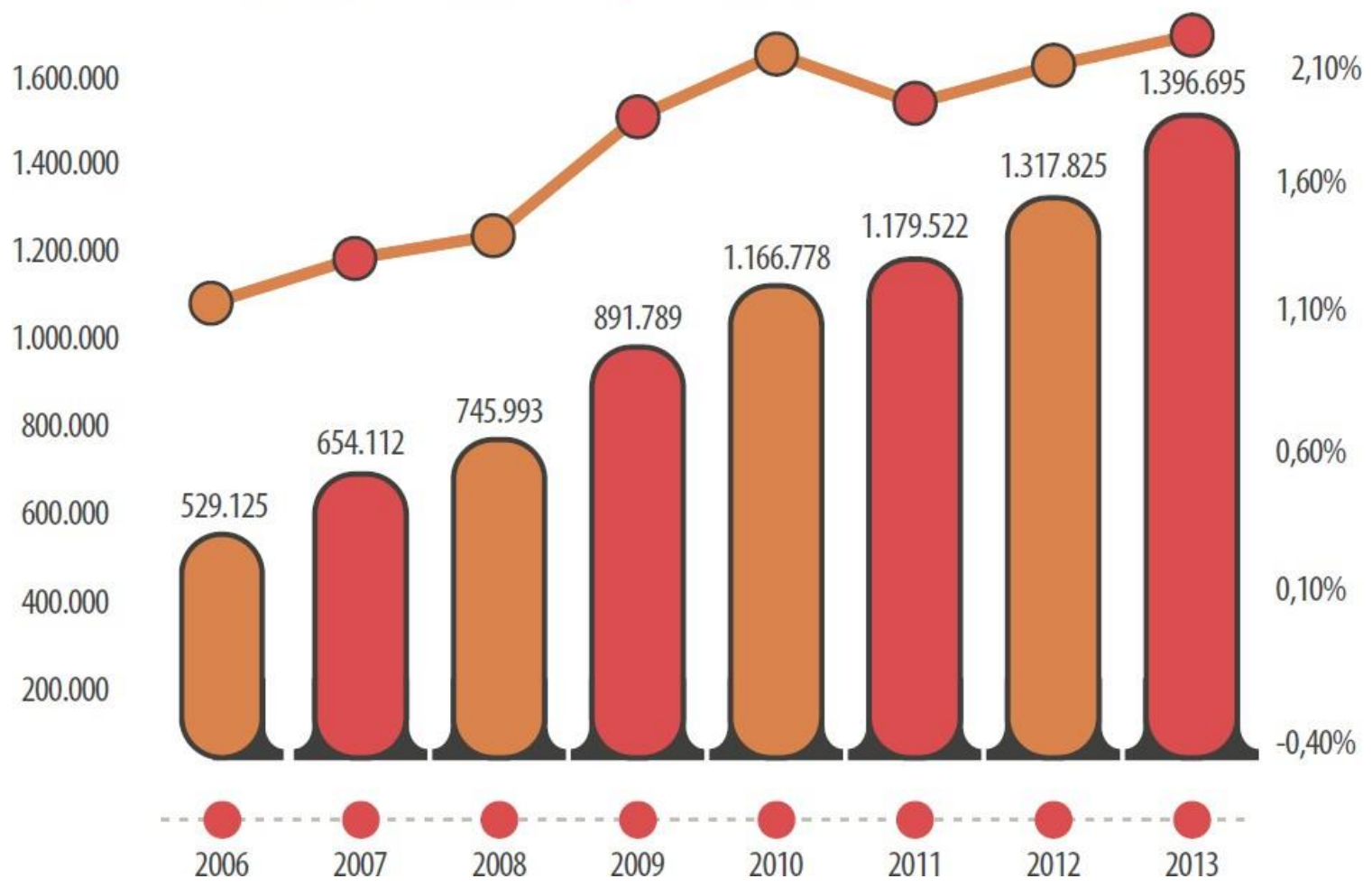

Figura 2. Gasto público de Ecuador en educación superior.

Fuente: (Ramírez Gallegos, 2013, p. 40).

\section{Discusión y conclusiones}

La evolución de la investigación va enmarcada en un desarrollo de la investigación por descubrimiento la cual genera soluciones rápidas con el fin de satisfacer las necesidades básicas. Este conocimiento adquirido pasó de generación en generación, donde cada una pudo agregar mejoras y condiciones a dichos conocimientos. 
La investigación fue creando importancia en la sociedad. A medida que se fomentaba el conocimiento, se determinó que debería de tener una metodología descriptiva con el fin de obtener resultados más precisos y así evitar las ambigüedades y falsas indicaciones.

Con esta metodología se empezó a generar paradigmas dentro de la sociedad haciendo que las investigaciones fuesen hechas por personas calificadas para tales objetivos, lo cual excluye cualquier conocimiento, opinión y sugerencia de miembros de los sectores de la sociedad.

Este rol de investigador científico fue tomado por los docentes en las instituciones de educación superior creando líneas de investigación que en la gran mayoría se pierden en las paredes de las universidades sin fomentar el desarrollo del conocimiento y excluyendo los conocimientos de los demás miembros de la comunidad como lo son los técnicos de laboratorios, obreros, administrativos y estudiantes. La sociedad universitaria tiene un conglomerado de conocimientos adquiridos por años de servicio y que pueden ser de vital importancia para el desarrollo de cualquier investigación.

Esos conocimientos pueden trasmitirse de distintas formas, utilizando hasta herramientas digitales educativas donde se pueda conocer no solo las experiencias propias de la institución sino también conocer y analizar situaciones y experiencias de otras universidades incrementando el un crecimiento de la investigación y, aún más, el crecimiento del conocimiento.

Esta investigación participativa puede ser implantada en los miembros de los distintos consejos o directores universitarios como brazo ejecutor de planes nacionales de investigación, fomentado por el estado, con el fin de desarrollar conocimientos que sirvan para el crecimiento nacional, cultural y social del país. Se debe realizar una masificación de todos los aspectos, ya que, involucran un aumento de la matrícula y a su vez un aumento de personal, equipos y espacios para el desarrollo de la investigación lo que conlleva a un gran gasto público que se transformará en inversión cuando esas investigaciones sean llevadas al sector productivo de la nación.

\section{Referencias bibliográficas}

Carizo Estévez, J. D. (2010). Importancia de la investigación en la formación de pregrado. Panorama Cuba y Salud, 3. 
Gajardo, M. \&. (1983). Educación participativa: alternativas metodológicas. Revista latinoamericana de estudios educativos, 22.

Gallo, M. (2005). Masificación de la educación superior: una reflexión acerca de sus causas y contradicciones. Argentina: Centro de Documentación - Universidad Nacional del Mar del Plata.

Gil Pérez, D. (1994). Diez años de investigación en didácticas de la ciencia: Realizaciones y perspectivas. Investigación y experiencias didácticas, 11.

López Noguero, F. (2002). El análisis de contenido como método de investigación. Revista de Educación - Universidad de Huelva, 13.

Pereda, C. D. (1993). Investigación-Acción Participativa. Documentación Social, 1-2.

Pérez-Rubin, C. (2001). La creatividad y la inspiración intuitiva. Génesis y evolución de la investigación científica de los hemisferios cerebrales. Arte, Individuo y Sociedad, 16.

Rama, C. (2006). La Tercera Reforma de la Educación Superior en América Latina y el Caribe: masificación, regulaciones e internacionalización. Buenos Aires, Argentina: Informe sobre la Educación Superior en America Latina y el Caribe: 2000-2005.

Ramírez Gallegos, R. (2013). TERCERA OLA DE TRANSFORMACIÓN DE LA EDUCACIÓN SUPERIOR EN ECUADOR. Quito, Ecuador: Secretaria Nacional de Educación Superior, Ciencia, Tecnología e Innovación.

Sánchez, E. (2007). La investigación latinoamericana de la comunicación y su entorno social: Notas para una agenda. Guadalajara, México.

Valcárcel, M. (2006). Genesis y evolución del concepto y enfoques sobre el desarrollo. Lima, Perú: Departamento de Ciencias Sociales- Pontificia Universidad Católica del Perú.

Wittrock, M. (1989). Handbook of Research on Teaching. Madrid, España: Ediciones Paidós Ibérica, S.A. 